\title{
Relation between stress heterogeneity and aftershock rate in the rate-and-state model
}

\author{
Agnès Helmstetter ${ }^{1,2}$ and Bruce E. Shaw ${ }^{1}$ \\ Received 29 September 2005; revised 22 February 2006; accepted 2 March 2006; published 21 July 2006.
}

[1] We estimate the rate of aftershocks triggered by a heterogeneous stress change, using the rate-and-state model of Dieterich. We show that an exponential stress distribution $P_{\tau}(\tau) \sim \exp \left(-\tau / \tau_{0}\right)$ gives an Omori law decay of aftershocks with time $\sim 1 / t^{p}$, with an exponent $p=1-A \sigma_{n} / \tau_{0}$, where $A$ is a parameter of the rate-and-state friction law and $\sigma_{n}$ is the normal stress. Omori exponent $p$ thus decreases if the stress "heterogeneity" $\tau_{0}$ decreases. We also invert the stress distribution $P_{\tau}(\tau)$ from the seismicity rate $R(t)$, assuming that the stress does not change with time. We apply this method to a synthetic stress map, using the (modified) scale invariant " $k$ " slip model (Herrero and Bernard). We generate synthetic aftershock catalogs from this stress change. The seismicity rate on the rupture area shows a huge increase at short times, even if the stress decreases on average. Aftershocks are clustered in the regions of low slip, but the spatial distribution is more diffuse than for a simple slip dislocation. Because the stress field is very heterogeneous, there are many patches of positive stress changes everywhere on the fault. This stochastic slip model gives a Gaussian stress distribution but nevertheless produces an aftershock rate which is very close to Omori's law, with an effective $p \leq 1$, which increases slowly with time. We obtain a good estimation of the stress distribution for realistic catalogs when we constrain the shape of the distribution. However, there are probably other factors which also affect the temporal decay of aftershocks with time. In particular, heterogeneity of $A \sigma_{n}$ can also modify the parameters $p$ and $c$ of Omori's law. Finally, we show that stress shadows are very difficult to observe in a heterogeneous stress context.

Citation: Helmstetter, A., and B. E. Shaw (2006), Relation between stress heterogeneity and aftershock rate in the rate-and-state model, J. Geophys. Res., 111, B07304, doi:10.1029/2005JB004077.

\section{Introduction}

[2] Much progress has been made in describing earthquake behavior based on the predictions of rate-and-state friction. The rate-and-state model explains the $1 / t$ decay of aftershock rate as a function of the time $t$ since the main shock (Omori's law) independent of the main shock magnitude, the scaling of aftershock duration with stressing rate, and the slow diffusion of aftershocks with time [Dieterich, 1994]. This success led several authors to provide timedependent earthquake probabilities using this model [Toda et al., 1998, 2005; Toda and Stein, 2003]. Many other physical mechanisms have been proposed to explain Omori law, such as subcritical crack growth [Das and Scholz, 1981; Shaw, 1993], viscous relaxation [Mikumo and Miyatake, 1979], static fatigue [Scholz, 1968; Narteau et al., 2002], postseismic slip [Schaff et al., 1998], or pore

\footnotetext{
${ }^{1}$ Lamont-Doherty Earth Observatory, Columbia University, Palisades, New York, USA.

${ }^{2}$ Now at Laboratoire de Géophysique Interne et Tectonophysique, Université Joseph Fourier, Grenoble, France.
}

Copyright 2006 by the American Geophysical Union. 0148-0227/06/2005JB004077\$09.00 fluid flow [Nur and Booker, 1972]. The rate-and-state model of Dieterich [1994] is probably the best candidate, however, because it only relies on a rate-and-state-dependent friction law observed in laboratory experiments.

[3] At the same time, a number of fundamental puzzles remain. One of the most striking is the abundance of aftershocks on the rupture surface, where indeed most aftershocks occur. This is in stark contrast with simple pictures of the rupture process, which suggest stress should have decreased on the rupture surface and there should therefore be a dearth of aftershocks there. A second fundamental puzzle concerns the time dependence of aftershocks. Here, subtle but significant deviations from an inverse time decay of the rate of aftershocks is seen in averages of aftershock rates [Helmstetter et al., 2005]. While Dieterich [1994] explained this as a consequence of the spatial dependence of stress as it decreases away from the fault, or as a change of stressing rate with time, such mechanisms do not seem to properly explain the aftershocks occurring on the rupture area. Thus both the spatial and temporal distribution of the majority of aftershocks have yet to be fully explained. Here, we show how an extension of the rate-and-state formulation, which takes as its foundation a heterogeneous stress field, can explain these observations. 


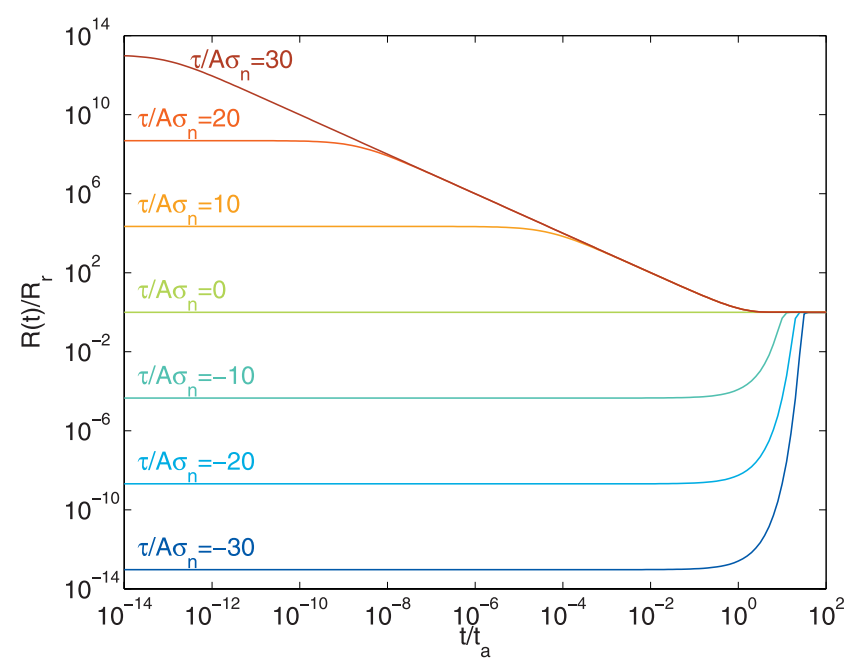

Figure 1. Seismicity rate $R(t, \tau)$ (normalized by the reference seismicity rate $R_{r}$ ) as a function of time (normalized by the aftershock duration $t_{a}$ ), given by the rate-andstate model (3) with a uniform stress step, for different values of the stress change, ranging from $\tau / A \sigma_{n}=-30$ (bottom) to $\tau / A \sigma_{n}=30$ (top).

We then use this model to estimate stress heterogeneity from aftershock rates.

[4] Our work builds off of the pioneering work of Dieterich [1994], who derived a relation between seismicity rate and stress history, for a population of faults obeying rate-and-state friction. For a uniform positive stress step, the rate-and-state model gives an Omori law decay of the seismicity rate $R(t) \sim t^{-p}$ with $p=1$ for intermediate times. At very short times, smaller than a characteristic time $c$, which depends on the stress change, the seismicity rate is constant. Dieterich [1994] also computed the aftershock rate for a dislocation, with a uniform stress decrease on the rupture area, and a positive stress change outside the rupture, decaying as $\tau \sim 1 / \sqrt{r}$ in the near field, and $\tau \sim 1 / r^{3}$ in the far field for $r \gg L$. As distance from the fault increases, the characteristic time $c$ increases. Integrating over the fault, the seismicity rate approximately obeys Omori law $R(t) \sim 1 / t^{p}$, with an apparent exponent $p<1$.

[5] Dieterich et al. [2000, 2003] used the rate-and-state model of seismicity to invert stress history from seismicity rate, and apply this method to Hawaii seismicity. They discretize the space, with a grid size of about $1 \mathrm{~km}$, and assume that the stress is uniform in each cell. This method then gives the stress history in each cell. The assumption that the stress is uniform at scales of a few $\mathrm{km}$ is reasonable for the stress change induced by a dyke intrusion, as in [Dieterich et al., 2000, 2003], or for the coseismic stress change induced by a large earthquake in the far field. However, the coseismic stress change on the main shock fault plane, where most aftershocks occur, is probably very heterogeneous at all scales [Herrero and Bernard, 1994].

[6] In this paper, we investigate how heterogeneity of the Coulomb stress change and of the normal stress modifies the temporal decay of aftershocks with time, both on the fault and off the fault. We assume that the stress changes instantaneously after the main shock, and we neglect the relaxation of stress on the fault due to aseismic slip or viscous relaxation. We also neglect the stress change, and seismicity rate change, induced by aftershocks. We then try to invert for the stress distribution on the fault plane from the aftershock rate, using the rate-and-state model, and assuming the main source of heterogeneity is the coseismic stress change.

\section{Relation Between Stress Distribution and Seismicity Rate}

[7] Dieterich [1994] derives a differential equation which gives the seismicity rate $R(t, \tau)$ as a function of the stress history $\tau(t)$. His model assumes an infinite population of faults which obeys rate and state friction, with the same properties for all faults.

[8] The state variable $\gamma$ is related to the stress $\tau$ by

$$
\partial \gamma=\frac{1}{A \sigma_{n}}[\partial t-\gamma \partial \tau],
$$

where $\tau$ is the "modified" Coulomb stress change [Dieterich et al., 2000], and $\sigma_{n}$ is the normal stress. The state variable $\gamma$ is a function of the seismicity rate $R(t, \tau)$

$$
R(t, \tau)=\frac{R_{r}}{\gamma(t, \tau) \dot{\tau_{r}}},
$$

where $R_{r}$ is the steady state seismicity rate at the reference stressing rate $\dot{\tau}_{r}$. From laboratory experiments, coefficient $A$ generally has values between 0.005 and 0.02 , for various temperature and pressure conditions [Dieterich, 1994].

[9] Dieterich [1994] used expression (1) to derive the seismicity rate $R(t, \tau)$ triggered by a single stress step $\tau$. We assume that stress rate after the stress step is constant $d \tau / d t=$ $\dot{\tau}_{r}$, and that the seismicity rate before the main shock is equal to the reference seismicity rate $R_{r}$. Using (1), the seismicity rate following the stress step is

$$
R(t, \tau)=\frac{R_{r}}{\left(e^{-\tau / A \sigma_{n}}-1\right) e^{-t / t_{a}}+1},
$$

where $t_{a}$ is the duration of the aftershock sequence

$$
t_{a}=\frac{A \sigma_{n}}{\dot{\tau}_{r}} .
$$

This relation (3) is illustrated in Figure 1 for different values of the stress change. For each positive stress value, the seismicity rate is constant for $t \ll t_{a} e^{-\tau / A \sigma_{n}}$, and then decreases with time for $t_{a} e^{-\tau / A \sigma_{n}} \ll t \ll t_{a}$ according to Omori law with an exponent $p=1$. For a negative stress change, the seismicity rate decreases after the main shock. In both cases, the seismicity rate recovers its reference value $R=R_{r}$ for $t \gg t_{a}$. The goal of this work is to extract the stress distribution from the seismicity rate. This is a difficult problem, because, as shown in Figure 1, the seismicity rate does not depend on the stress change over a relatively large time interval. 
[10] For a heterogeneous stress field $\tau(\vec{r})$, with a distribution (probability density function) $P_{\tau}(\tau)$, the seismicity rate integrated over space is

$$
\begin{gathered}
R(t)=\int R(t, \tau(\vec{r})) d \vec{r} \\
R(t)=\int_{-\infty}^{\infty} R(t, \tau) P_{\tau}(\tau) d \tau \\
R(t)=\int_{0}^{\infty} R(t, c) P_{c}(c) d c
\end{gathered}
$$

where $c=t_{a} e^{-\tau / A \sigma_{n}}$ is a characteristic time of the aftershock rate, such that $R(t, c) \sim 1 / c$ for $t \ll c$ and $R(t, c) \sim 1 / t$ for $c \ll t \ll t_{a}$.

[11] Equation (7) is a Fredholm integral equation of the first kind. It has, at most, one solution [Riele, 1985]. Equation (7) has a simple approximate solution in the case when the stress change has an exponential distribution

$$
P_{\tau}(\tau) \sim e^{-\tau / \tau_{0}},
$$

where $\tau_{0}$ is a positive scaling stress parameter, which characterizes the width of the stress distribution. This corresponds to a power law distribution of "corner times" $c$

$$
P(c)=P_{\tau}(\tau) \frac{d \tau}{d c}=c^{-1+A \sigma_{n} / \tau_{0}} .
$$

We also consider an approximate expression for the seismicity rate (3) valid for short times $t \ll t_{a}$

$$
R(t, \tau) \approx \frac{R_{r}}{e^{-\tau / A \sigma_{n}}+t / t_{a}}=\frac{R_{r} t_{a}}{c+t} .
$$

Substituting (9) and (10) in (7), we get

$$
R(t)=\int_{0}^{\infty} \frac{R_{r} t_{a} c^{-1+A \sigma_{n} / \tau_{0}}}{c+t} d c \sim \frac{1}{t^{1-A \sigma_{n} / \tau_{0}}},
$$

Expression (11) corresponds to Omori law with an exponent

$$
p=1-\frac{A \sigma_{n}}{\tau_{0}} .
$$

Because equation (6) has at most one solution, the exponential stress distribution is the only distribution which produces a pure Omori law decay for $t \ll t_{a}$, without any cutoff or crossover at short times. However, other distributions, e.g., a Gaussian, produce aftershock rate that is very close to Omori's law, over a very large time range. The stress distribution for small or negative values is not constrained by the seismicity rate at short times $t \ll t_{a}$, so deviations from an exponential for negative stresses do not produce deviations from Omori law at short times.

[12] Expression (12) shows that Omori exponent depends on stress heterogeneity. The parameter $\tau_{0}$ represents the

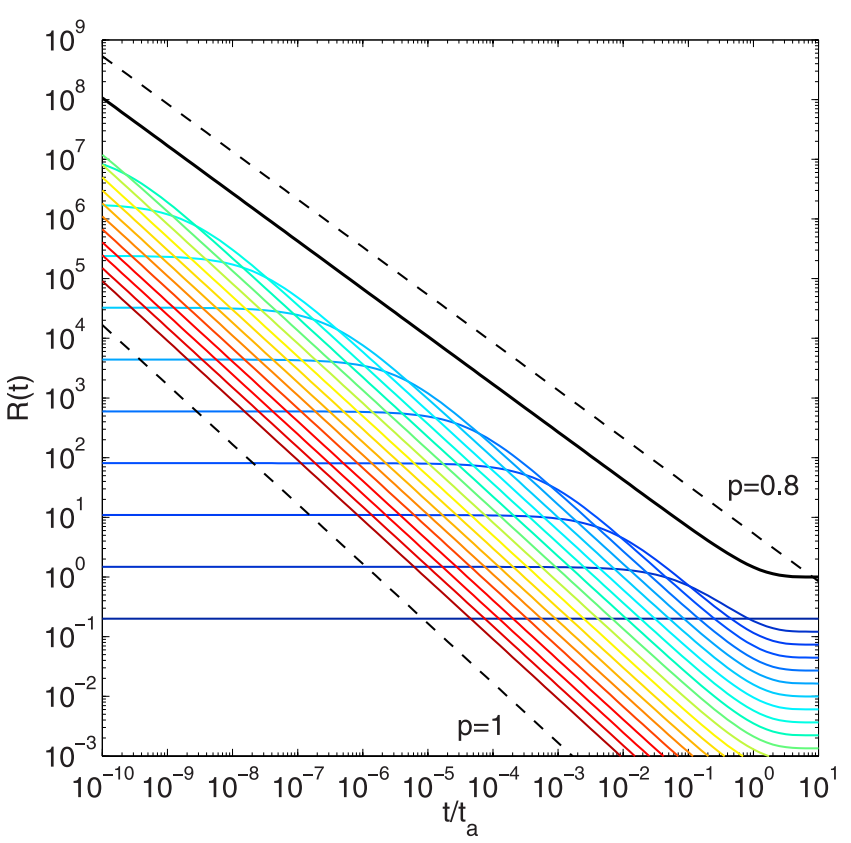

Figure 2. Seismicity rate $R(t, \tau)$ for a uniform stress change $\tau$ (thin colored lines), ranging from $\tau=0$ (blue flat curve) to $\tau=50 \mathrm{MPa}$ (red curve), weighted by the probability $P_{\tau}(\tau)$, using $A \sigma_{n}=1 \mathrm{MPa}$. The stress distribution is given by $P_{\tau}(\tau) \sim \exp (-\tau / 5)$ with $\tau>0$. The solid black line is the total seismicity rate $R(t)=$ $\int_{0}^{\infty} R(t, \tau) P_{\tau}(\tau) d \tau$. The superposition of curves $R(t, \tau)$ with a power law distribution of crossover times $c=t_{a}$ $\exp \left(-\tau / A \sigma_{n}\right)$ gives rise to a power law decay of $R(t)$ with an exponent $p \approx 0.8$. The dashed lines are Omori laws with $p=1$ (bottom) and $p=0.8$ (top).

width of the stress distribution for $\tau>0$. The more heterogeneous the stress is (larger $\tau_{0}$ ), the larger $p$ is (closer to 1 ). Figure 2 illustrates how the rate-and-state model with a heterogeneous stress distribution produces a power law decay with an exponent $p<1$.

[13] Helmstetter et al. [2005] found that for stacked aftershock sequences in Southern California, Omori exponent is close to 0.9 , for times ranging between a minute (but possibly even less) and one year, and for main shock magnitudes between 2 and 7.5. This suggests that the stress distribution is close to exponential in the tail, with a characteristic stress $\tau_{0} \approx 10 A \sigma_{n}$. Assuming that $A=0.01$ (as measured in laboratory friction experiments [Dieterich, 1994]) and $\sigma_{n}=100 \mathrm{MPa}$ (corresponding to the lithostatic pressure at a depth of about $5 \mathrm{~km}$ ), this gives $A \sigma_{n}=1 \mathrm{MPa}$ and $\tau_{0}=10 \mathrm{MPa}$, a value larger than the typical stress drop $\sigma_{0}=3 \mathrm{MPa}$ [Ide and Beroza, 2001], but of the same order of magnitude. However, a few studies tried to estimate $A \sigma_{n}$ directly from earthquake catalogs, and obtained values smaller than the ones derived from the laboratory value of A. Dieterich [1994] found $A \sigma_{n}=\sigma_{0} / 20$, from the relation between aftershock duration and the recurrence time (assuming characteristic earthquakes). This gives $A \sigma_{n}=0.15 \mathrm{MPa}$ assuming a stress drop of $3 \mathrm{MPa}$. Cochran et al. [2004] used the rate-and-state model to model tidal triggering of earthquakes, and obtained a preferred value of $A \sigma_{n}=0.064 \mathrm{MPa}$, and an acceptable range $0.048<A \sigma_{n}<0.11 \mathrm{MPa}$. 
[14] The rate-and-state model with a uniform stress step (3) cannot explain an Omori law decay with $p>1$. Equation (7) does not have a solution with $P_{\tau}(\tau)>0$ and $t \ll t_{a}$ in this case. Some aftershock sequences, however, have an Omori exponent larger than one. The only solution in order to obtain a $p$ value larger than one in the rate-and-state model is to have a variation of stress with time, which may be due to postseismic slip or viscous relaxation [Dieterich, 1994]. Other explanations for Omori's law do allow for larger $p$ values [Mikumo and Miyatake, 1979; Shaw, 1993; Narteau et al., 2002].

\section{Estimating the Stress Distribution From Aftershock Rate}

[15] We have shown above that according to the rate-andstate model, the Omori exponent provides some information on the stress heterogeneity (but only if $p<1$ ). Furthermore, we can (in theory) obtain the complete stress distribution (in the region where we measure the seismicity rate) from the temporal evolution of the seismicity rate. Expression (6) indeed provides a method for estimating the full distribution $P_{\tau}(\tau)$, provided we observe the seismicity rate $R(t)$ over a wide enough time interval.

[16] We first discretize the integration over stress and times, using a linear sampling for stress, and a logarithmic sampling for times, using the same number $N$ of points. Equation (6) is then similar to the system of $N$ linear equations

$$
R\left(t_{j}\right)=\sum_{i=1}^{N} R\left(t_{j}, \tau_{i}\right) P_{\tau}\left(\tau_{i}\right)\left(\tau_{i+1}-\tau_{i}\right) .
$$

We divide both sides of equation (13) by $R\left(t_{j}\right)$ to stabilize the problem. Equation (13) thus becomes

$$
1=\sum_{i=1}^{N} \frac{R\left(t_{j}, \tau_{i}\right)}{R(t)} P_{\tau}\left(\tau_{i}\right)\left(\tau_{i+1}-\tau_{i}\right)=M \times P,
$$

where $M$ is a $N \times N$ matrix $M(i, j)=\left(\tau_{i+1}-\tau_{i}\right) R\left(t_{j}, \tau_{i}\right) / R\left(t_{j}\right)$ and the vector $P$ is the stress distribution at points $\tau_{1}, . ., \tau_{N}$.

[17] The inversion of the stress distribution from (14) is an ill-posed problem, i.e., the solution is very sensitive to noise. We thus use the regularization method of Riele [1985]. We introduce an additional constraint to (13), minimizing either the first derivative $\left|P^{\prime}(\tau)\right|$, the smoothness $\left\|P^{\prime \prime}(\tau)\right\|$, or the distance between $P_{\tau}(\tau)$ and an initial guess $P_{0}(\tau)$. (e.g., a Gaussian distribution). Instead of solving directly (14), we minimize the quantity

$$
\|M P-1\|^{2}+\alpha\|L(P)\|^{2},
$$

where $\alpha>0$ is the regularization parameter, and $L$ is a linear operator, e.g., $L(P)=P-P_{0}, L(P)=P^{\prime}$ (first derivative), or $L(P)=P^{\prime \prime}$ (second derivative). We also impose that the stress distribution is positive. We thus search for the positive vector $P$ that minimizes equation (15), using the nonlinear least squares fitting program given by Lawson and Hanson [1974].
[18] In practice, the estimation of $P_{\tau}(\tau)$ for large $\tau$ is limited by the minimum time $t_{\min }$ at which we can reliably estimate the seismicity rate. The largest stress we can resolve is of the order of $\tau_{\max }=-A \sigma_{n} \log \left(t_{\min } / t_{a}\right)$. Practically, this time $t_{\min }$ may be as low as a few seconds, if we correct from catalog incompleteness shortly after the main shock (Z. Peng et al., Anomalous seismicity rate immediately before and after main shock rupture from highfrequency waveforms in Japan, submitted to Journal of Geophysical Research, 2006). For negative stress, we are limited by the maximum time $t_{\max }$ after the main shock, and by our assumptions that secondary aftershocks are negligible, and that the stress does not change with time (e.g., neglecting postseismic relaxation). In order to resolve $P_{\tau}(\tau)$ for negative values, we need to know the seismicity rate for times larger than the aftershock duration $t_{a}$ (i.e., usually at least a few years). Indeed, the seismicity rate after a stress decrease is close to zero for $t \ll t_{a}$, so that the measure of $R(t)$ for $t \ll t_{a}$ does not provide any information on $P_{\tau}(\tau)$ for $\tau<0$.

\section{Application of the Method to a Stochastic Slip Model}

\subsection{Stochastic $\boldsymbol{k}^{2}$ Slip Model}

[19] We have tested the rate-and-state model on a realistic synthetic slip pattern. Herrero and Bernard [1994] proposed a kinematic, self-similar model of earthquakes. They assumed that the slip distribution at small scales, compared to the rupture length $L$, does not depend on $L$. This led to a slip power spectrum for high wave number equal to

$$
u(k)=C \frac{\sigma_{0}}{\mu} \frac{L}{k^{2}} \text { for } k>1 / L,
$$

where $\sigma_{0}$ is the stress drop (typically $3 \mathrm{MPa}$ ), $\mu$ is the rigidity (typically $3300 \mathrm{MPa}$ in the lower crust), and $C$ is a shape factor close to 1 . For wavelengths larger than the rupture length $L$, the power spectrum is constant

$$
u(k)=C \frac{\sigma_{0}}{\mu} L^{3} \text { for } k<1 / L .
$$

This model (16) reproduces the $1 / f^{2}$ power spectrum of seismograms for large frequencies [Herrero and Bernard, 1994].

\subsection{Shear Stress Change and Seismicity Rate on the Fault}

[20] We have used the $k^{2}$ model to generate a synthetic slip pattern, and compute the shear stress change on the fault from the slip [Andrews, 1980; Ripperger and Mai, 2004]. Note that the seismicity rate given by (3) depends on the Coulomb stress change, which is equal to the shear stress change on the fault because the normal stress change on a planar fault is zero. If we analyze off-fault aftershocks or complex rupture geometries, we would have to consider changes in normal stress as well.

[21] We have modified the $k^{2}$ model in order to have a finite standard deviation of the stress distribution. The $k^{2}$ model (16) produces a shear stress change with a power spectrum $\tau(k) \sim k^{-1}$ for large $k$, because the stress is 

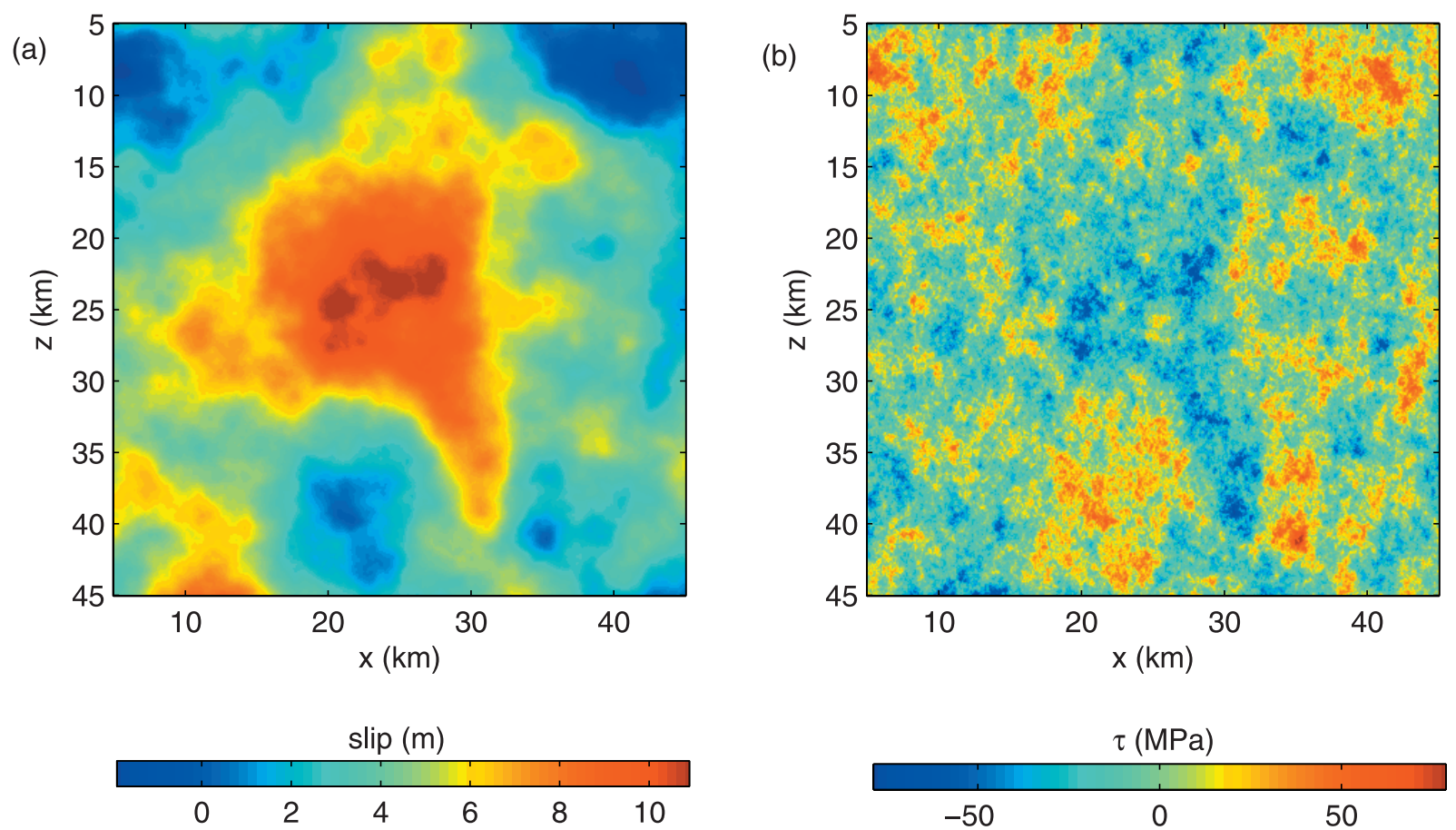

Figure 3. (a) Stochastic slip model, with a power spectrum $u(k)=1 /(k L+1)^{2.3}$ (where $L=50 \mathrm{~km}$ is the rupture length), a stress drop $\sigma_{0}=3 \mathrm{MPa}$, and a cell size $d x=0.1 \mathrm{~km}$. (b) Shear stress change (parallel to the slip direction).

approximately the derivative of the slip. As a consequence, the shear stress change for the $k^{2}$ model is extremely heterogeneous, with an infinite standard deviation. The exponent $n=2$ in the $k^{2}$ model (16) is thus a minimum physical value for the slip power spectrum [Herrero and Bernard, 1994]. Using $u(k) \sim k^{-2}$ produces a shear stress change with a standard deviation which diverges logarithmically as the maximum wave number increases. Thus Omori $p$ value for this slip model tends to 1 as the grid resolution increases. We have thus replaced the exponent $n=$ 2 in (16) by $n=2.3$, and smoothed the crossover at $k=1 / L$, using

$$
u(k)=C \frac{\sigma_{0}}{\mu} \frac{L^{3}}{(k L+1)^{n}} .
$$

[22] We have computed the stress change on the fault from this synthetic slip model, for a fault of $50 \times 50 \mathrm{~km}$, with a resolution $d x=0.1 \mathrm{~km}$, and a stress drop $\sigma_{0}=3 \mathrm{MPa}$ (i.e., the average stress change on the fault is $-3 \mathrm{MPa}$ ). The maps of the slip and stress on the fault are shown in Figure 3. The stress field has large variations, from about -90 to $90 \mathrm{MPa}$, due to slip variability. We did not constrain the slip to be positive. This could be done by changing the phase of the lowest mode, and tapering the slip close to the edges, so that the maximum slip is at the center [Herrero and Bernard, 1994]. Doing so introduces small deviations of the stress distribution from a Gaussian distribution for $\tau \approx 0$ but does not introduce significant changes on the seismicity rate.

[23] We have then estimated the seismicity rate on the fault predicted by the rate-and-state model, by integrating numerically (5) using the observed stress map, and $A \sigma_{n}=$ $1 \mathrm{MPa}$. While the stress on average decreases on the fault, the seismicity rate shows a huge increase after the main shock (by a factor $10^{10}$, but, of course, the seismicity rate at short times, smaller than the duration of the earthquake, has no physical sense) (see Figure 4). It then decays with time approximately according to Omori law, with an apparent exponent $p=0.93$. At large

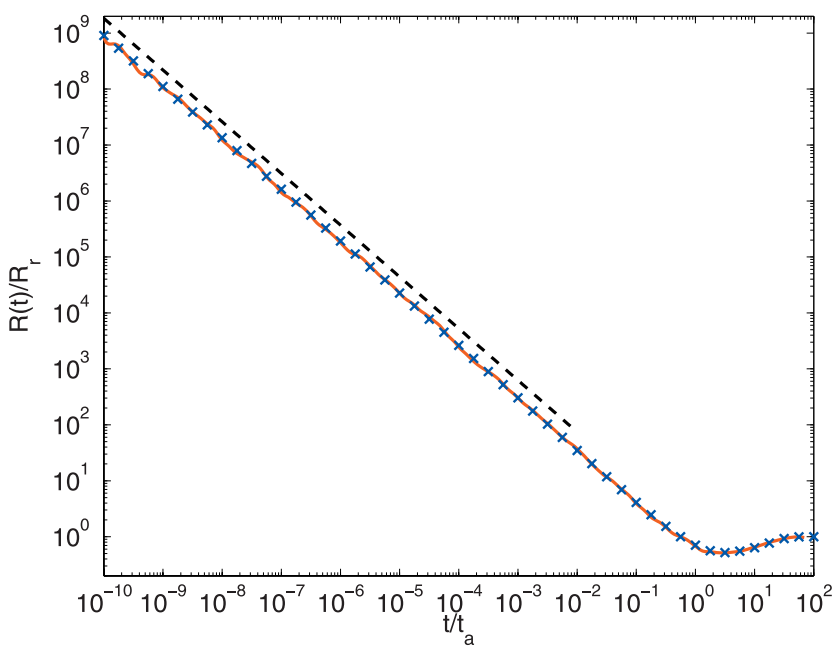

Figure 4. Seismicity rate given by the rate-and-state model [Dieterich, 1994], for the stress change shown in Figure 3, assuming $A \sigma_{n}=1 \mathrm{MPa}$, and without earthquake interactions. The solid red line is the seismicity rate estimated from the simulated earthquake catalog (see model 1 in Table 1). The dashed black line is a fit by Omori's law for $t<t_{a} / 100$, with exponent $p=0.93$. The crosses show the fit with the rate-and-state model assuming a Gaussian $P_{\tau}(\tau)$. 
Table 1. Parameters of the Synthetic Aftershock Catalogs ${ }^{\mathrm{a}}$

\begin{tabular}{|c|c|c|c|c|c|c|c|c|c|c|c|}
\hline Catalog & $N$ & $t_{\min }$ & $t_{\max }$ & $p$ & $-\sigma_{0}$ & $A \sigma_{n}$ & $-\sigma_{0, g} / A \sigma_{n}$ & $\tau^{*}$ & $\tau^{*}{ }_{g} / A \sigma_{n}$ & $t_{a}$ & $t_{a, g}$ \\
\hline 1 & 154,447 & $10^{-10}$ & 100. & 0.924 & -3.01 & 1.0 & -3.19 & 19.6 & 19.5 & $10^{7}$ & $1.00 \times 10^{7}$ \\
\hline 2 & 3,550 & $10^{-6}$ & 1. & 0.938 & -3.01 & 1.0 & -3.14 & 19.6 & 20.6 & $10^{7}$ & $0.95 \times 10^{7}$ \\
\hline $3^{b}$ & 392 & $10^{-6}$ & 1. & 0.929 & -3.01 & 1.0 & -9.45 & 19.6 & 20.8 & $10^{7}$ & $1.58 \times 10^{7}$ \\
\hline $3^{\mathrm{b}}$ & 392 & $10^{-6}$ & 1. & 0.929 & -3.01 & 1.0 & -1.19 & 19.6 & 18.3 & $10^{7}$ & $1.00 \times 10^{7 \mathrm{c}}$ \\
\hline $3^{\mathrm{b}}$ & 392 & $10^{-6}$ & 1. & 0.929 & -3.01 & 1.0 & $-3.01^{\mathrm{c}}$ & 19.6 & 18.7 & $10^{7}$ & $1.12 \times 10^{7}$ \\
\hline 4 & 231 & $10^{-5}$ & 0.1 & 0.948 & -3.01 & 1.0 & -57.5 & 19.6 & 42.8 & $10^{7}$ & $5.01 \times 10^{7}$ \\
\hline 4 & 231 & $10^{-5}$ & 0.1 & 0.948 & -3.01 & 1.0 & $-3.01^{\mathrm{c}}$ & 19.6 & 24.1 & $10^{7}$ & $0.90 \times 10^{7}$ \\
\hline 4 & 231 & $10^{-5}$ & 0.1 & 0.948 & -3.01 & 1.0 & -6.03 & 19.6 & 25.8 & $10^{7}$ & $1.00 \times 10^{7 \mathrm{c}}$ \\
\hline 5 & 203,998 & $10^{-10}$ & 100. & 0.995 & -3.01 & 0.1 & -30.8 & 19.6 & 195. & $10^{7}$ & $1.00 \times 10^{7 \mathrm{c}}$ \\
\hline 6 & 3,857 & $10^{-6}$ & 1. & 0.992 & -3.01 & 0.1 & -45.9 & 19.6 & 133. & $10^{7}$ & $1.21 \times 10^{7}$ \\
\hline 6 & 3,857 & $10^{-6}$ & 1. & 0.992 & -3.01 & 0.1 & -29.2 & 19.6 & 171. & $10^{7}$ & $1.00 \times 10^{7 \mathrm{c}}$ \\
\hline 6 & 3,857 & $10^{-6}$ & 1. & 0.992 & -3.01 & 0.1 & $-30.1^{\mathrm{c}}$ & 19.6 & 125. & $10^{7}$ & $1.09 \times 10^{7}$ \\
\hline
\end{tabular}

${ }^{a}$ Number $N$ of events, time interval $\left[t_{\min } t_{\max }\right]$, Omori exponent $p$ (measured by maximum likelihood from the simulated catalog for $t / t_{a}<0.01$ ), average stress change $-\sigma_{0}$, value of $A \sigma_{n}$ used for the simulations, and standard deviation $\tau^{*}$ (in MPa), and results of the inversion: $\sigma_{0, g}$, $\tau_{g}^{*}$, and $t_{a, g}$, estimated assuming a Gaussian stress distribution $P_{\tau}(\tau)$. Stress values are in $\mathrm{MPa}$.

${ }^{\mathrm{b}}$ This catalog is a subset of catalog 2 , obtained by increasing the minimum magnitude by one unit.

${ }^{\mathrm{c}}$ The parameter was fixed to its real value in the inversion.

times $t \approx t_{a}$, the seismicity rate decreases below its reference rate due to the negative stress values.

[24] Marsan [2006] reached similar conclusions, using the same model: the main effect of stress heterogeneity on the fault is to produce a short-term increase of the seismicity rate, and to delay the seismic quiescence on the fault by months to years.

\subsection{Synthetic Aftershock Catalog}

[25] We have generated synthetic earthquake catalogs according to the rate-and-state model, using the (modified) $k^{2}$ model (18) to generate the stress change. We have simulated aftershock sequences triggered by this heterogeneous stress change, without including earthquakes interactions (i.e., without coseismic stress changes induced by aftershocks), using the method of Dieterich et al. [2003]. We assume a nonstationary Poisson process with an average seismicity rate $R(t, \tau)$ given by (3). We generate aftershocks in each cell independently of the other cells, assuming that the stress is uniform in each cell. We do not need to generate event magnitudes, because we do not include secondary aftershocks in our simulation. We consider that each aftershock does not modify the stress field or the seismicity rate.

[26] In each cell, we generate events one after the other. If the last event in the cell occurred at a time $t_{i}$ after the main shock, the probability that the next earthquake will occur at a time smaller than $t_{i}+d t$ is given by

$$
F\left(d t, t_{i}\right)=\exp \left[-\int_{t_{i}}^{t_{i}+d t} R\left(t^{\prime}, \tau\right) d t^{\prime}\right] .
$$

The function $F\left(d t, t_{i}\right)$ increases from 0 to 1 as $d t$ ranges from 0 to $\infty$. To determine the time $t_{i+1}=t_{i}+d t$ of the next event, we generate a random number $z$ between 0 and 1, and we solve for $F\left(d t, t_{i}\right)=z$. We have generated six synthetic catalogs from the stress field shown in Figure 3, using $A \sigma_{n}=1 \mathrm{MPa}$ or $A \sigma_{n}=$ $0.1 \mathrm{MPa}$. We used different values of the reference rate $R_{r}$, and of time interval $t_{\min }-t_{\max }$ (see Table 1), in order to test how the inversion method depends on the quality of the catalog.

[27] Figure 5 shows a seismicity map, for a synthetic catalog generated with $A \sigma_{n}=1 \mathrm{MPa}$. Aftershocks are clustered in the regions of low slip, where the stress has increased after the main shock. This pattern is very different from simple dislocation models, which produce a uniform stress drop on the rupture area. Therefore these simple slip models produce aftershocks only around the rupture area. In contrast, the complex slip model shown in Figure 3 produces many spots of stress increase on the rupture area. It thus explains why, in real data, most aftershocks are located on or very close to the rupture area.

4.4. Inversion of Stress History From Seismicity Rate

[28] We have first applied the method of Dieterich et al. $[2000,2003]$ on this synthetic stress field shown in Figure 3b. Dieterich et al. [2000, 2003] estimate the stress history $\tau(t)$ at any point on a grid, assuming that the stress change is homogeneous in each cell, but may change with time. The stress history is obtained from the seismicity rate

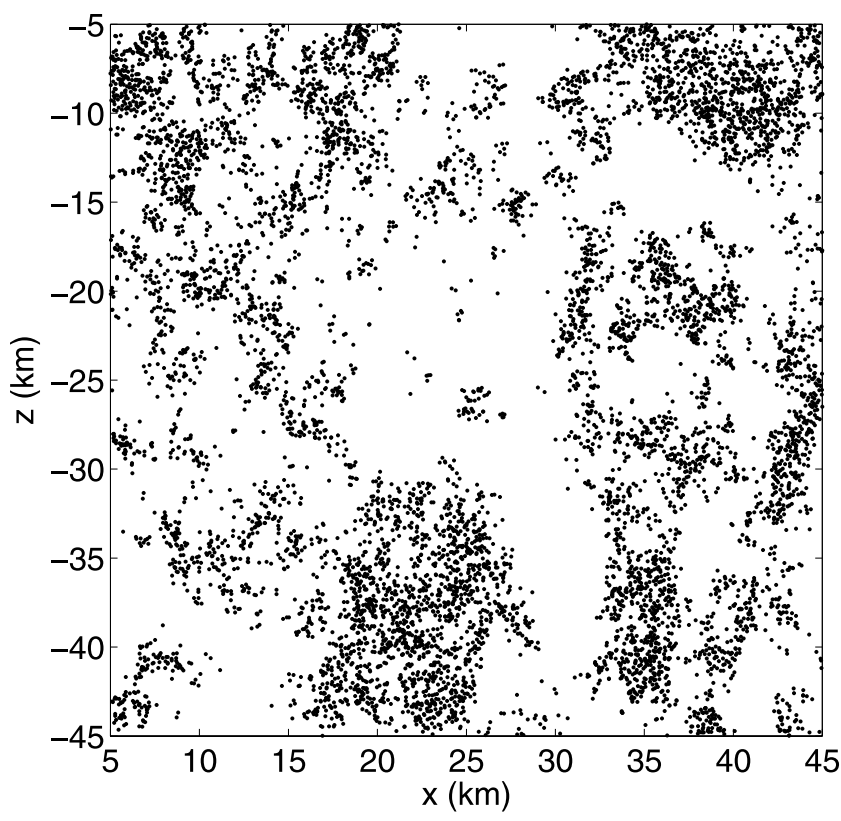

Figure 5. Seismicity map, for a synthetic catalog generated using the rate-and-state model [Dieterich, 1994], for the stress change shown in Figure 3, assuming $A \sigma_{n}=1 \mathrm{MPa}$, and without earthquake interactions. Only events with $t<t_{a}$ are shown. 


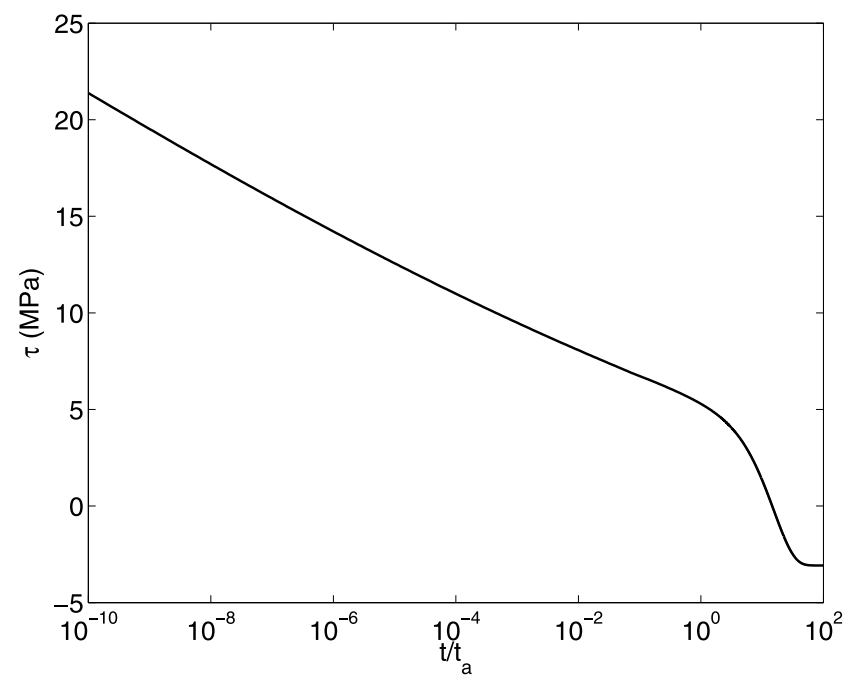

Figure 6. Shear stress change, as a function of the time after the main shock, estimated from the seismicity rate using Dieterich et al.'s [2000, 2003] method. We solved equation (1) for the stress history, assuming that the stress is uniform in space but changes with time.

by solving equation (1). We wanted to apply this method on this synthetic stress model to test how stress heterogeneity affects the inverted stress change. The results are shown in Figure 6 . The inverted stress change at short times is close to the maximum stress change $\approx 100 \mathrm{MPa}$, and then decreases down to a value close to the average stress change $\approx-3 \mathrm{MPa}$ at large times $t>t_{a}$. Dividing the fault into smaller size cells would not improve the results very much. Because this slip model is self-similar, there are almost everywhere some parts of the fault where the stress (and thus the seismicity rate) increases. This shows that a smallscale stress heterogeneity, without any time dependence, is interpreted by this method as a variation of stress with time. Also, it shows that a stress decrease cannot be resolved if it is mixed with a stress increase, unless looking at very long times. This may explain why stress shadows are so difficult to observe [Felzer and Brodsky, 2005].

\subsection{Inversion of Stress Distribution From Seismicity Rate}

[29] This test shows that variability with time is hard to distinguish from small-scale heterogeneity in space based on the temporal evolution of the seismicity rate.

[30] In order to characterize the coseismic stress change on the fault plane, we thus need to neglect one effect (smallscale heterogeneity) or the other (time variation). Our method estimates the stress distribution on the fault from the seismicity rate, assuming that stress does not change with time. In theory (if we had an infinite time interval, a huge number of aftershocks, no foreshocks or secondary aftershocks, and if we knew the parameters $R_{r}, t_{a}$, and $A \sigma_{n}$ ), this method provides the distribution of stress on the fault. If the fault is divided into smaller cells, this method gives a map of the average stress change in each cell, as well as its variability.

[31] For each synthetic catalog, we have measured the seismicity rate on the fault by smoothing aftershock times.
We used a kernel method to estimate $R(t)$ from aftershocks time $t_{i}$, with $i=1$ to $N$, with a $\operatorname{lognormal}$ filter

$$
R(t)=\sum_{i=1}^{N} \frac{1}{h t \sqrt{2 \pi}} \exp \left(-\frac{\left(\log _{10}(t)-\log _{10}\left(t_{i}\right)\right)^{2}}{2 h^{2}}\right)
$$

with a kernel width $h=0.08$.

[32] We then used the inversion method described previously to estimate the stress distribution $P_{\tau}(\tau)$ from the seismicity rate. We used the regularization condition $L(P)=$ $P^{\prime}$ in (15), i.e., minimizing the derivative of $P_{\tau}(\tau)$, using $\alpha=$ $10^{4}$ (decreasing $\alpha$ produces huge fluctuations of $P_{\tau}(\tau)$ ).

[33] We have also estimated the Gaussian stress distribution that best fits the observed seismicity rate. We evaluate the mean $-\sigma_{0}$, and the standard deviation $\tau^{*}$ of the Gaussian function, as well as the aftershock duration $t_{a}$, using a maximum likelihood approach. We maximize the $\log$ likelihood function defined by

$$
L=\sum_{i=1, N} \log R\left(t_{i}\right)-\int_{t_{\min }}^{t_{\max }} R(t) d t,
$$

where the seismicity rate is given by

$$
R(t)=\int_{-\infty}^{\infty} R(t, \tau) \frac{e^{-\left(\tau+\sigma_{0}\right)^{2} / 2 \tau^{* 2}}}{\tau^{*} \sqrt{2 \pi}} d \tau .
$$

The log likelihood function is maximized when the rate estimate $R$, weighted logarithmically, is large when events occur at times $t_{i}$, and when the total rate estimate integrated over time is low.

[34] Table 1 gives the parameters of each simulation, and the results of the inversion. Figures 7 and 8 show the real stress distribution (evaluated from the stress map shown in Figure $3 b$ ) and the inverted one, for each synthetic after-

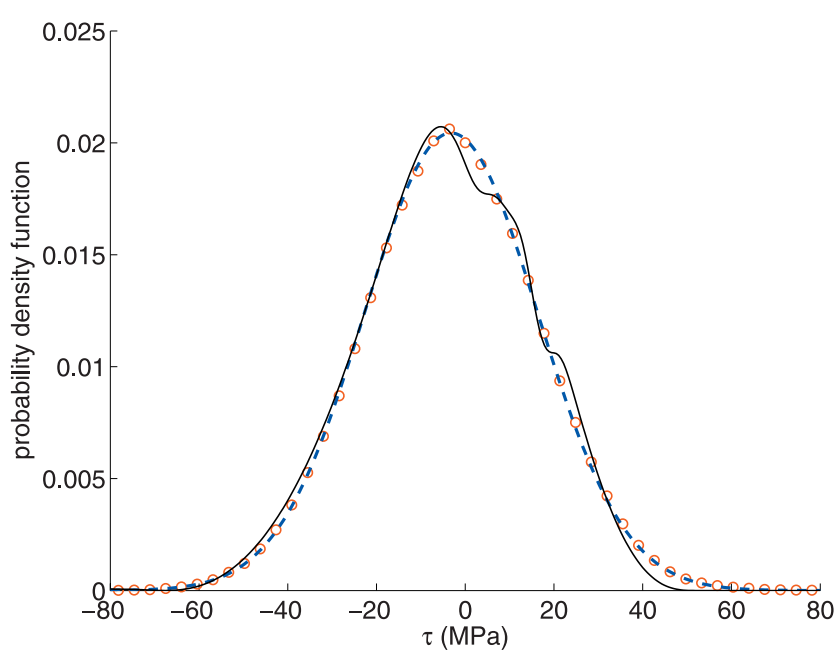

Figure 7. Stress distribution estimated directly from the stress map shown in Figure 3b (circles) and inverted from the seismicity rate shown in Figure 4. The solid black line is the solution of equation (15)). The best fitting Gaussian distribution is shown as a blue dashed line. 


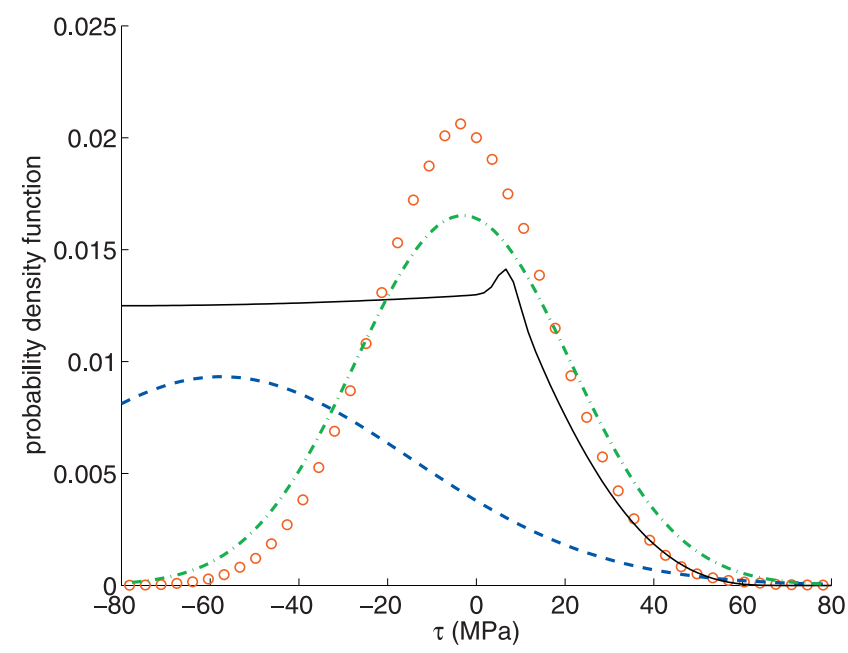

Figure 8. Stress distribution estimated directly from the stress map shown in Figure 3b (circles) and inverted from the seismicity rate shown in Figure 9 (see model 4 in Table 1). The solid black line is the solution of equation (15). The best fitting unconstrained Gaussian distribution is shown as a blue dashed line. The green dash-dotted line shows the best fitting Gaussian with fixed stress drop.

shock catalog. We test both inversion methods, either solving (15) for $P_{\tau}(\tau)$ for $80<\tau<80 \mathrm{MPa}$, or assuming a Gaussian stress distribution.

[35] Figure 4 compares the theoretical seismicity rate given by (5) using the observed stress field, with the seismicity rate estimated from the seismicity catalog using (20), and with the reconstructed seismicity rate estimated using (6) from the inverted stress distribution. For this synthetic catalog, the seismicity rate is almost indistinguishable from an Omori law with an exponent $p=$ 0.93 for $t / t_{a}<10$.

[36] In the first 2 catalogs in Table 1, with more than several thousands events, we obtain a very good estimation (error less than $6 \%$ ) on all parameters $\tau^{*}, \sigma_{0}$ and $t_{a}$. If the number of events decreases to 392 events, without changing the time interval, we still obtain a rather good estimation of $t_{a}$ and $\tau^{*}$, but the error on the stress drop increases (see model 3 in Table 1). For a shorter catalog (catalog 4 in Table 1), with 292 events and only 4 ranges of magnitude in time, the stress drop is not constrained, unless we fix the aftershock duration to its true value. Alternatively, we can fix the stress drop and obtain a rather good estimation of $\tau^{*}$ and $t_{a}$. This shows that the main effect in recovering $\sigma_{0}, \tau^{*}$ and $t_{a}$ is the catalogue time interval, which needs to extend over a reasonable fraction of $t_{a}$. This is because very different values of $t_{a}$ and $\sigma_{0}$ can produce very similar seismicity rate $R(t)$ for $t<t_{a} / 100$, as can be shown in Figure 9. If we decrease $A \sigma_{n}$, keeping $\tau^{*}$ fixed, the Omori exponent becomes closer to 1 , and the error on all parameters increases (see models 5 and 6).

[37] When inverting for the complete distribution $P_{\tau}(\tau)$, the results are pretty good for the first simulation, with an unrealistic large time interval and number of events. There are, however, deviations in the tails, for $\tau>30 \mathrm{MPa}$, which correspond to very short corner times $c=t_{a} \exp \left(-\tau / A \sigma_{n}\right)=$ $10^{-13}$, much smaller than the minimum time $t_{\min } / t_{a}=10^{-10}$ used for the inversion of $P_{\tau}(\tau)$. For catalogs $2-4$ in Table 1, the distribution of $P_{\tau}(\tau)$ is not constrained for $\tau<0$, and for $\tau \gg 1$, because of the limited time interval. The results are very poor for both simulations 5 and 6 in Table 1, with $A \sigma_{n}=$ $0.1 \mathrm{MPa}$ and Omori exponent $p=0.993$. In this case, we have almost no resolution on $P_{\tau}(\tau)$ for $\tau<0$. This method only provides a rough estimate of the width of the distribution for $\tau>0$. Thus, in practice, unless one has a very long catalogue in time, and significant deviations from Omori law, little can be said about the stress shadow regions.

\subsection{Off-Fault Aftershocks}

[38] We can make simple estimates of the stress change and seismicity rate off of the fault plane. For mode III rupture, static elasticity reduces to a Laplacian $\Delta u=0$. For a Laplacian, a Fourier mode with wave number $k$ along an infinite fault decays exponentially into the bulk proportional to $k$ times the distance $y$ to the fault. With these basis functions, we can easily extrapolate off of the fault, although since it neglects rupture end effects, it is valid only for distances less than the rupture length $L$ and in areas along side the main shock rupture area, and not extending into the lobes of increasing stress beyond the finite rupture length. Thus we are looking at regions which would be in the stress "shadow" of a simple rupture. Within this region, at a distance $y<L$ from the fault, the power spectrum of the displacement for the modified slip model (18) becomes $u(k, y)$ $\sim(k L+1)^{-n} \exp (-k y)$. The power spectrum of the stress change is given by

$$
\tau(k, y) \sim k u(k, y) \sim \frac{e^{-k y}}{(k L+1)^{n-1}} .
$$

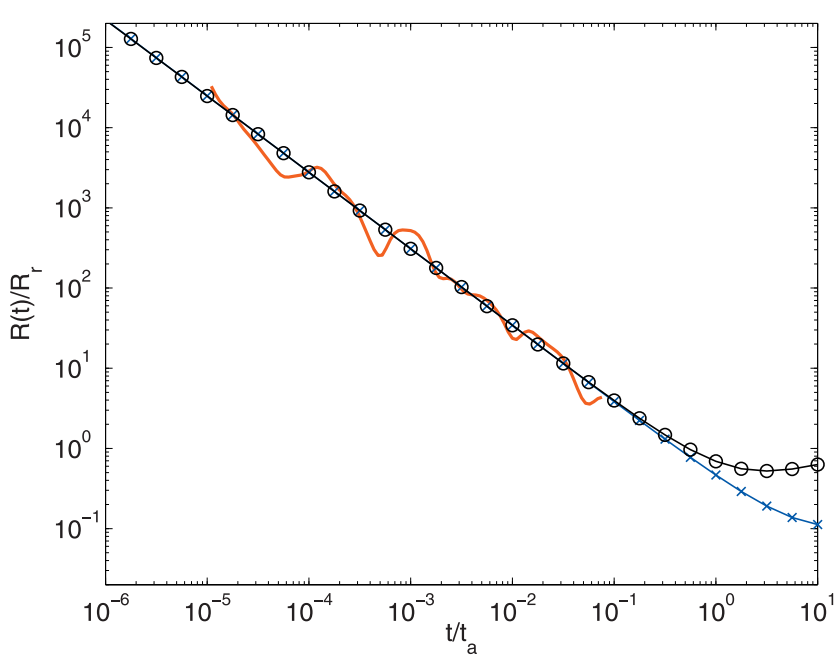

Figure 9. Seismicity rate given by the rate-and-state model [Dieterich, 1994], for the stress change shown in Figure 3, assuming $A \sigma_{n}=1 \mathrm{MPa}$, and without earthquake interactions. The solid red line is the seismicity rate estimated from the simulated earthquake catalog (see catalog 4 in Table 1). The crosses show the fit with the rate-and-state model assuming a Gaussian $P_{\tau}(\tau)$ and inverting for $t_{a}, \sigma_{0}$, and $\tau^{*}$. The circles are the fit assuming a Gaussian $P_{\tau}(\tau)$ with the stress drop fixed to its real value. 

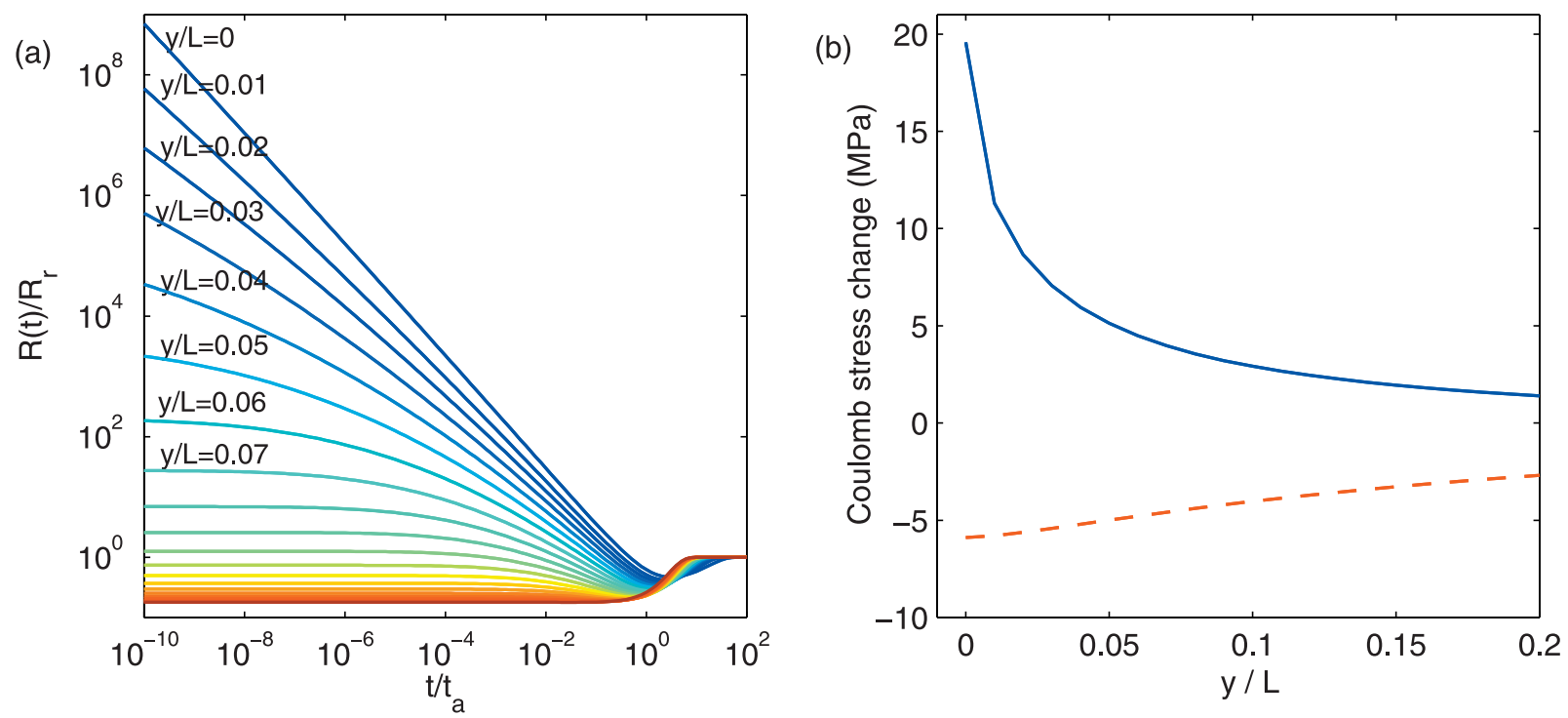

Figure 10. (a) Seismicity rate for different values of the distance to the fault $y / L$ decreasing from $y / L=0$ (top) to $y / L=0.2$ (bottom), using the slip model shown in Figure $3 \mathrm{a}$, and assuming $A \sigma_{n}=1 \mathrm{MPa}$. (b) Standard deviation (solid line) and absolute value of the mean (dashed line, the average stress change is always negative) of the stress distribution as a function of the distance to the fault.

This shows how the stress heterogeneity decays very rapidly with distance from the rupture surface.

[39] Figure 10a shows the seismicity rate for different values of the distance from the fault $y / L$, using the slip model shown in Figure $3 \mathrm{a}$. We computed the stress at a distance $y$ from the fault using $\tau(k, y)=\tau(k, 0) \exp (-k y)$, i.e., multiplying the stress map shown in Figure $3 \mathrm{~b}$ by $\exp (-k y)$ in the Fourier domain. The stress distribution is reasonably close to a Gaussian distribution at all distances. Therefore we have used the best fitting Gaussian distribution in order to compute the seismicity rate shown in Figure 10a. The standard deviation of the stress distribution decreases very fast with the distance to the fault, which produces a strong drop of the seismicity rate off of the fault. The average stress decreases much slower with $y$. Figure $10 \mathrm{~b}$ shows the falloff with distance of these quantities. For $y / L>0.1$, the stress field is much more homogeneous and mostly negative (the standard deviation is smaller than the absolute mean stress). Therefore the seismicity rate for $y / L>$ 0.1 is smaller than the reference rate at all times $t<t_{a}$. Marsan [2006] also used the rate-and-state model to investigate how stress change heterogeneity modifies the rate of off-fault aftershocks. He used a slightly different slip model, and assumed the spatial dependence of the stress variability decayed with the same form as the stress, as distance cubed. With this assumption he found, not surprisingly, larger distances of triggering.

[40] In practice, it is difficult to analyze the rate of offfault aftershocks, because the aftershock rate and the reference seismicity rate decrease with the distance from the fault, and because of location errors. Also, secondary aftershocks triggered by off-fault events will perturb the stress field and seismicity rate with additional stress heterogeneity. Our seismicity rate estimates here presume focal mechanisms of aftershocks similar to the main shock focal mechanism; other focal mechanisms could have different rates, but optimally oriented plane estimates may not be the best approach [McCloskey et al., 2003]. In any case, we do see very rapid falloff of the seismicity with distance from the fault, a point which deserves further observational exploration. Note that Figure 10a shows the seismicity rate normalized by the reference rate $R_{r}$. If $R_{r}$ decreases with the distance to the fault, the decrease of the aftershock rate with $y$ will be even faster than shown in Figure 10a.

\subsection{Gaussian Versus Exponential Stress Distribution}

[41] While the pure Omori law with $p<1$ occurs for the exponential distribution of stress changes, we find numerically that a Gaussian stress distribution (which the $k^{2}$ model and many other models give), also gives realistic looking $p$ values over wide ranges of timescales. Some insight into why this is the case can be gained by noting that for a sufficiently wide range of values, a Gaussian is a good enough approximation of an exponential. Taking the ratio of a Gaussian to an exponential

$$
\begin{aligned}
& \exp \left(-\frac{\left(\tau+\sigma_{0}\right)^{2}}{2 \tau^{* 2}}\right) / \exp \left(\frac{-\tau}{\tau_{0}}\right) \\
& =\exp \left[-\frac{1}{2}\left(\frac{\tau+\sigma_{0}}{\tau^{*}}-\frac{\tau^{*}}{\tau_{0}}\right)^{2}-\frac{\sigma_{0}}{\tau_{0}}+\frac{\tau^{* 2}}{2 \tau_{0}^{2}}\right]
\end{aligned}
$$

For $\tau / \tau^{*}=\tau^{*} / \tau_{0}-\sigma_{0} / \tau^{*} \pm 1$ this is within a factor $\exp (1)$ of being constant. Thus, over an $e$-folding range of $\tau^{*} / \tau_{0}$ we have something well approximated by an exponential.

[42] We can use this result to obtain an approximate analytical expression for the effective Omori exponent produced by a Gaussian stress distribution. Expression (24) shows that the exponential distribution closer to the Gaussian one for a stress $\tau$ has a characteristic parameter $\tau_{0}=\tau^{*} /\left(\sigma_{0}+\tau\right)$. As Figure 2 illustrates, the more important contribution to the aftershock rate at time $t \ll$ $t_{a}$ is due to stress values of the order of $\tau_{c}=-A \sigma_{n} \log \left(t / t_{a}\right)$. If the stress change obeys a Gaussian distribution, stresses 


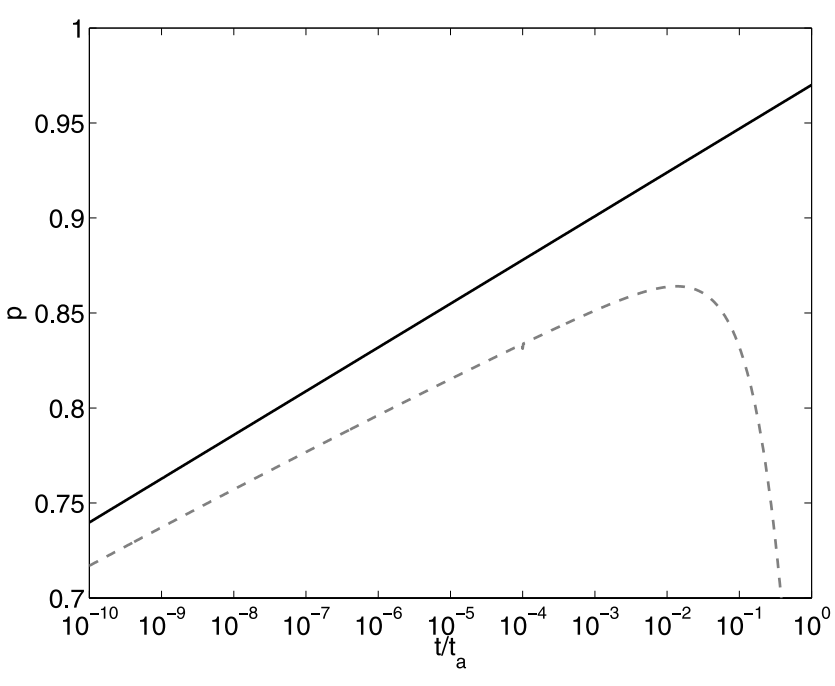

Figure 11. Variation of the effective Omori exponent with time, for a Gaussian stress distribution of mean $-\sigma_{0}=$ $-3 \mathrm{MPa}$ and standard deviation $\tau^{*}=10 \mathrm{MPa}$. The dashed line is the exact solution (given by integrating numerically (6)), and the solid line is the approximate analytical solution (26).

larger than $\tau_{c}$ are less frequent than for $\tau=\tau_{c}$, therefore they have a smaller contribution to the seismicity rate at time $t$. Smaller stress values $\tau<\tau_{c}$ are more frequent, but the seismicity rate at time $t$ is negligible compared to larger stress values. We thus obtain the following relation between the parameters $\sigma_{0}$ and $\tau^{*}$ of a Gaussian distribution, and the parameter $\tau_{0}$ of the exponential distribution which better explains the aftershock rate at a given time $t$

$$
\tau_{0}=\frac{\tau^{* 2}}{\sigma_{0}-A \sigma_{n} \log \left(t / t_{a}\right)} .
$$

Using expression (12), we obtain the following relation between the effective Omori exponent at time $t$ and the parameters $\sigma_{0}$ and $\tau^{*}$ of a Gaussian stress distribution

$$
p \approx 1-\frac{A \sigma_{n} \sigma_{0}-A^{2} \sigma_{n}^{2} \log \left(t / t_{a}\right)}{\tau^{2}},
$$

showing the slow increase of $p$ with time. Figure 11 compares this approximate solution (26) with the variation of $p$ with time computed by integrating numerically (6), using $A \sigma_{n}=1 \mathrm{MPa}$, for a Gaussian stress distribution with $\sigma_{0}=3 \mathrm{MPa}$ and $\tau^{*}=10 \mathrm{MPa}$. The approximate solution (26) for Omori exponent is quite good for short times $t \ll t_{a}$, but the difference with the exact solution increases as time approaches $t_{a}$. This expression (26) also shows us the inherent tradeoff between the mean stress change $-\sigma_{0}$ and the variance of the stress change $\tau^{*}$ in affecting the $p$ value.

\section{Discussion}

[43] We have considered above only heterogeneity of the Coulomb stress change. However, there are other important factors that affect the temporal evolution of the seismicity rate, such as heterogeneity of the friction law parameter $A$, normal stress, and stressing rate, multiple interactions between aftershocks, foreshocks, and postseismic relaxation.

\subsection{Heterogeneity of the Friction Parameter $A$, Normal Stress, Stressing Rate, and Reference Seismicity Rate}

[44] We have shown that Coulomb stress change heterogeneity modifies the temporal evolution of the seismicity rate, compared to a uniform stress change. However, other kinds of heterogeneity may also impact the aftershock decay with time, in particular the normal stress. Normal stress heterogeneity enters the problem in two ways, through the "modified" Coulomb stress change $\tau$, and through the $A \sigma_{n}$ term in the denominator.

[45] Slip on a rough fault will produce coseismic changes of the normal stress [Dieterich, 2005]. For coseismic changes of the normal stress which are small compared to the normal stress, we can assume that $A \sigma_{n}$ does not change with time, and account for coseismic changes of $\sigma_{n}$ only in the coseismic Coulomb stress change $\tau$. For larger coseismic changes of normal stress, we have to use a more complex form for the relation (1) between stress history and seismicity rate [Dieterich, 1994], and equation (3) is no more valid.

[46] In addition to coseismic stress changes of $\sigma_{n}$, there are also spatial fluctuations of $A \sigma_{n}$. For instance, we expect both $A$ and $\sigma_{n}$ to change with depth. With a wide variety of materials making up fault zones and the presence of fluids, there is probably no lower bound on $A \sigma_{n}$. The first effect of introducing heterogeneity of $A \sigma_{n}$ is to increase the fluctuations of the normalized stress $\tau / A \sigma_{n}$, i.e., the standard deviation of $\tau / A \sigma_{n}$ is larger than $\tau^{*} /\left\langle A \sigma_{n}\right\rangle$. Therefore Omori exponent increases if $A \sigma_{n}$ is more heterogeneous. Neglecting the fluctuations of $A \sigma_{n}$ will thus overestimate $\tau^{*}$.

[47] The second effect is to introduce fluctuations of the aftershock duration $t_{a}$, which scales with $A \sigma_{n}$. Fluctuation of $A \sigma_{n}$ thus decrease the apparent aftershock duration. The value of $t_{a}$, inverted assuming $\tau / A \sigma_{n}$ is uniform, is smaller than its average value $t_{a}$. Also, $A \sigma_{n}$ heterogeneity widens the duration of the quiescence (time period when $R(t)<R_{r}$ ).

[48] We have illustrated the effect of normal stress heterogeneity in Figure 12, which compares the seismicity rate with and without fluctuations of $A \sigma_{n}$. Fluctuations of coseismic Coulomb stress changes are modeled by a Gaussian distribution of mean $-\sigma_{0}=-3 \mathrm{MPa}$ and standard deviation $\tau^{*}=5 \mathrm{MPa}$. For $A \sigma_{n}$, we use a lognormal distribution of mean $\left\langle A \sigma_{n}\right\rangle=1 \mathrm{MPa}$ and standard deviation $\operatorname{std}\left(A \sigma_{n}\right)=7.3 \mathrm{MPa}$. The main effects of $A \sigma_{n}$ heterogeneity is to increase the apparent Omori exponent (measured for $t<0.01 \mathrm{yr})$ from 0.44 to 0.91 , and to decrease the apparent aftershock duration, (defined as the time when the aftershock rate decreases below its reference value) from 0.2 to $0.05 \mathrm{yr}$.

[49] Inverting for the Coulomb stress distribution from $R(t)$, assuming that $A \sigma_{n}=1 \mathrm{MPa}$ everywhere, gives $\tau^{*}{ }_{g}=$ 15.7 MPa, $\sigma_{0, g}=9.9 \mathrm{MPa}$ and $t_{a, g}=0.15 \mathrm{yr}$, instead of the true value $\tau^{*}=5 \mathrm{MPa}, \sigma_{0, g}=3 \mathrm{MPa}$ and $\left\langle t_{a}\right\rangle=1 \mathrm{yr}$. The errors in the inverted parameters $\tau_{g}^{*}, \sigma_{0, g}, t_{a, g}$ are negligible when the Coulomb stress change $\tau$ is more heterogeneous than $A \sigma_{n}$, i.e., if $\tau^{*} \gg \operatorname{std}\left(A \sigma_{n}\right)$. The fit of the aftershock rate with $A \sigma_{n}$ assumed constant gives a reasonably good fit to the seismicity rate computed including $A \sigma_{n}$ heterogeneity. 


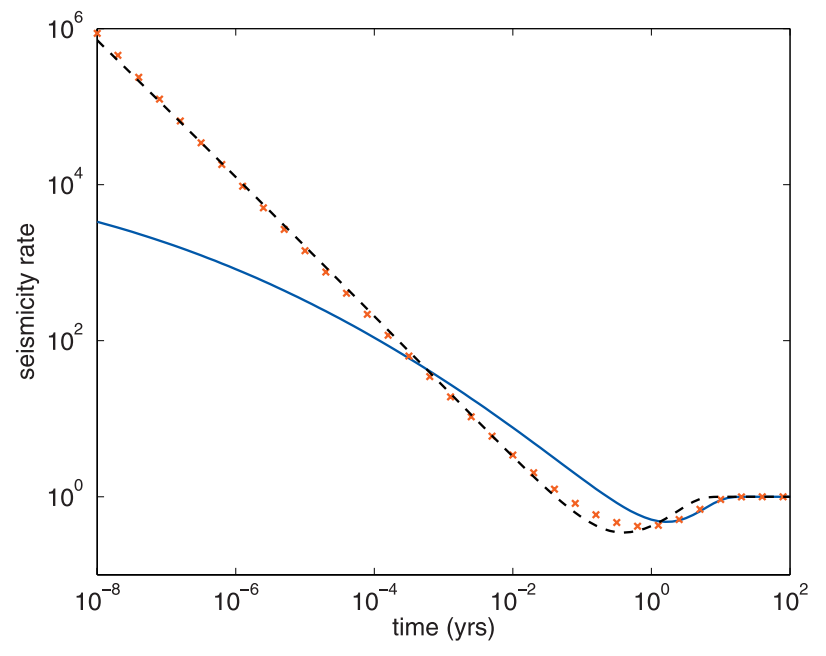

Figure 12. Seismicity rate for a Gaussian stress distribution $\left(\sigma_{0}=-3 \mathrm{MPa}, \tau^{*}=5 \mathrm{MPa}\right)$, without (solid line) and with (crosses) heterogeneity of $A \sigma_{n}$. Normal stress fluctuations are modeled by a lognormal distribution of average $1 \mathrm{MPa}$ and standard deviation $7.3 \mathrm{MPa}$. The dashed line is a fit by the rate-and-state model (3), assuming $A \sigma_{n}$ is uniform.

The misfit will probably be within the noise level for real data set. This shows that with the time dependence of the seismicity alone being the source of information, we cannot distinguish between heterogeneity of $\tau$ or $A \sigma_{n}$. Finding other effects which might be able to separate out these contributions of shear stress heterogeneity and normal stress heterogeneity remains an area worthy of further inquiry.

[50] The fact that $A \sigma_{n}$ heterogeneity increases the Omori exponent may explain why very low $p$ values are seldom observed, even outside the main shock rupture area, where Coulomb stress change is relatively uniform (see Figure 10b). This also explains why the crossover time $c$ for off-fault aftershocks is usually very short, as for on-fault aftershocks. It also makes stress shadows even more difficult to detect. Even in the regions where stress change is negative and not too heterogeneous $\left(\tau^{*}<\sigma_{0}\right)$, fluctuations of $A \sigma_{n}$ produce an increase of the seismicity rate at short times, while a uniform value of $A \sigma_{n}$ gives a quiescence at all times.

[51] Another parameter that affects the seismicity rate is the reference stressing rate, which modifies the aftershock duration $t_{a}$. Heterogeneity of the stressing rate will thus also yield an error in the inverted values of $\tau^{*}$ and $\sigma_{0}$. In contrast, the seismicity rate does not depend on the spatial fluctuations of the reference seismicity rate, but depends only on the average value of $R_{r}$. In practice, $R_{r}$ is measured from the average seismicity rate over a long time period before the main shock. The uncertainty on $R_{r}$ is generally of a factor of about 2. This could induce large relative errors on the stress drop estimate $\sigma_{0, g}$, but does not affect too much the inverted values of $\tau^{*}$ and $t_{a}$.

\subsection{Foreshocks}

[52] An assumption of our model is that the seismicity rate before the main shock is equal to the reference seismicity rate. However, most main shocks are preceded by foreshocks, so that the seismicity rate $R_{0}$ before the main shock is usually larger than the reference rate $R_{r}$. Using the results of Dieterich [1994], we can take into account this effect by replacing the term $e^{-\tau / A \sigma_{n}}$ in (3) by

$$
\frac{R_{r}}{R_{0}} \exp \left(-\frac{\tau}{A \sigma_{n}}\right)=\exp \left(-\frac{\tau}{A \sigma_{n}}-\log \left(\frac{R_{0}}{R_{r}}\right)\right) .
$$

The effect of increasing $R_{0}$ is thus equivalent to shifting the stress distribution toward larger values, by the amount $A \sigma_{n}$ $\log \left(R_{0} / R_{r}\right)$. Not correcting for this effect will thus overestimate the stress change.

\subsection{Secondary Aftershocks}

[53] We have neglected in this study the role of aftershocks in changing the seismicity rate and redistributing the stress. We know that most aftershocks may be secondary aftershocks, triggered by previous aftershocks [Felzer et al., 2003; Helmstetter and Sornette, 2003]. Ziv and Rubin [2003] studied a quasi-static fault model that is governed by rate- and state-dependent friction. They have shown that if the main shock is modeled as a uniform stress increase, the main effect of secondary aftershocks in the rate-andstate model is to renormalize the seismicity rate without changing its time dependence (i.e., without changing Omori $p$ value). If the stress change induced by the main shock is nonuniform, multiple interactions between earthquakes modify the spatial distribution of aftershocks [Ziv, 2003].

[54] Marsan [2006] also performed numerical simulations to model the effect of multiple interactions. He modeled the stress change induced by each aftershock by a Gaussian white noise of zero mean, i.e., assuming all aftershocks have the same size, and neglecting spatial correlation of the stress field. He concluded that the main effect of multiple interactions is to increase the reference rate, but also to decrease the ratio of the aftershock and background rates. The existence of multiple interactions also decreases the apparent aftershock duration, but does not change the Omori exponent.

[55] Therefore secondary aftershocks should not change the value of the width of the stress distribution inverted from the aftershock decay on the main shock fault, which is controlled by Omori exponent. However, multiple interactions may bias the value of the average stress change and aftershock duration. Developing more realistic models for multiple interactions remains an area worthy of further inquiry, but beyond the goals of this paper.

\section{Conclusion}

[56] We have shown how a new extension of the rate-andstate friction formulation for seismicity rates, which takes as its foundation a heterogeneous stress field, can explain the most prevalent and puzzling of aftershocks, those which occur on the main shock rupture area, where the stress decreases on average after the main shock. With this point of view, subtle but significant deviations from the pure inverse time Omori exponent are mapped onto measures of stress change heterogeneity on the fault. This contrasts with the established methodology of Dieterich et al. [2000, 2003], in which these deviations are mapped onto time dependent stress changes following the main shock.

[57] Taking the point of view that stress heterogeneity can be quite large at the local scale on the fault surface which ruptured, we have gained insights into a number of topics of relevance to stress heterogeneity's and earthquake behavior. 
Regarding stress shadows, we have seen how they are very difficult to detect in a heterogeneous stress context, relying on subtle details in the seismicity rates at times of order $t_{a}$, subtleties which would become even more difficult to detect if $t_{a}$ were nonuniform.

[58] Regarding stress heterogeneity relative to mean stress changes, we have found in our efforts to model seismicity changes with scale invariant slip distributions that typical stress changes are actually larger than mean stress drops on faults, so that a picture of a very rough stress distribution on a fault which has broken is a much better picture than standard crack-like models, which tend to concentrate aftershocks at the edges of ruptures. This provides important constraints on physical models of earthquakes. Finally, we have shown that modest catalogue lengths allow an accurate inversion for some stress heterogeneity parameters, if the only source of heterogeneity is the Coulomb stress change.

[59] However, there are probably other important factors that affect the temporal evolution of the seismicity rate, such as heterogeneity of the friction law parameter $A$, effective normal stress, and stressing rate, multiple interactions between aftershocks, and postseismic relaxation. In particular, heterogeneity of $A \sigma_{n}$ may explain why Omori exponent and characteristic time $c$ does not seem to depend on stress change amplitude [Felzer, 2005]. We have shown that earthquake triggering is not only controlled by the average values of the Coulomb stress change, or of the effective normal stress, but rather by their heterogeneity. Particularly, short time aftershock rate is mainly controlled by the maximum stress change in this region, rather than by its average value. Estimation of coseismic slip from seismograms or geodesy is not accurate enough to estimate smallscale variations of the stress change on the fault plane. Therefore we need to couple large-scale deterministic slip models with small-scale stochastic slip models, in order to reproduce the spatiotemporal distribution of triggered earthquakes. This also shows the difficulty of inverting the stress field from the spatiotemporal variations of the seismicity rate. Real data is limited in number of events, catalog duration, and location accuracy; and we have only rough estimates of the large-scale average value of the friction parameters, normal stress and stressing rate. With the limited information given by the seismicity rate, it is hard to characterize the different factors that control earthquake triggering, especially on or close to the fault, where stress and material properties are very heterogeneous.

[60] Acknowledgments. We thank Jim Dieterich, Fabrice Cotton, Michel Campillo, and Alon Ziv for useful discussions. Jeffrey McGuire (Associate Editor) and Susanna Gross (reviewer) also provided interesting suggestions that helped improve the quality of the manuscript. Part of this work was done while the authors were at the KITP in Santa Barbara. This research was supported in part by the National Science Foundation under grants PHY99-0794 and EAR03-37226, by the Southern California Earthquake Center (SCEC), and by the Brinson Foundation. SCEC is funded by NSF Cooperative Agreement EAR-0106924 and USGS Cooperative Agreement $02 \mathrm{HQAG} 0008$. This is SCEC contribution 930

\section{References}

Andrews, D. J. (1980), A stochastic fault model: 1. Static case, J. Geophys. Res., 85, 3867-3877.

Cochran, E. S., J. E. Vidale, and S. Tanaka (2004), Earth tides can trigger shallow thrust fault earthquakes, Science, 306, 1164-1166.
Das, S., and C. H. Scholz (1981), Theory of time-dependent rupture in the Earth, J. Geophys. Res., 86, 6039-6051.

Dieterich, J. (1994), A constitutive law for rate of earthquake production and its application to earthquake clustering, J. Geophys. Res., 99, $2601-$ 2618

Dieterich, J. H. (2005), Role of stress relaxation in slip of geometrically complex faults, Eos Trans. AGU, 86(52), Fall Meet. Suppl., Abstract NG31A-04.

Dieterich, J. H., V. Cayol, and P. Okubo (2000), The use of earthquake rate as a stress meter at Kilauea volcano, Nature, 408, 457-460.

Dieterich, J. H., V. Cayol, and P. Okubo (2003), Stress changes before and during the Puo-Kupaianaha eruption, U.S. Geol. Surv. Prof. Pap., 1676, $187 \mathrm{pp}$

Felzer, K. R. (2005), Evidence that stress amplitude does not affect the temporal distribution of aftershocks, Eos Trans. $A G U, 86(52)$, Fall Meet Suppl., Abstract S11C-05.

Felzer, K. R., and E. E. Brodsky (2005), Testing the stress shadow hypothesis, J. Geophys. Res., 110, B05S09, doi:10.1029/2004JB003277.

Felzer, K. R., R. E. Abercrombie, and Goran Ekström (2003), Secondary aftershocks and their importance for aftershock prediction, Bull. Seismol. Soc. Am., 93, 1433-1448.

Helmstetter, A., and D. Sornette (2003), Importance of direct and indirect triggered seismicity in the ETAS model of seismicity, Geophys. Res. Lett., 30(11), 1576, doi:10.1029/2003GL017670.

Helmstetter, A., Y. Kagan, and D. Jackson (2005), Importance of small earthquakes for stress transfers and earthquake triggering, J. Geophys. Res., 110, B05S08, doi:10.1029/2004JB003286.

Herrero, A., and P. Bernard (1994), A kinematic self-similar rupture process for earthquakes, Bull. Seismol. Soc. Am., 84, 1216-1228.

Ide, S., and G. C. Beroza (2001), Does apparent stress vary with earthquake size?, Geophys. Res. Lett., 28, 3349-3352.

Lawson, C. L., and R. J. Hanson (1974), Solving Least Squares Problems, Prentice-Hall, Inc., Upper Saddle River, N. J.

Marsan, D. (2006), Can coseismic stress variability suppress seismicity shadows?, Insights from a rate-and-state friction model, J. Geophys. Res., 111, B06305, doi:10.1029/2005JB004060.

McCloskey, J., S. S. Nalbant, S. Steacy, C. Nostro, O. Scotti, and D. Baumont (2003), Structural constraints on the spatial distribution of aftershocks, Geophys. Res. Lett., 30(12), 1610, doi:10.1029/2003GL017225.

Mikumo, T., and T. Miyatake (1979), Earthquake sequences on a frictional fault model with non-uniform strengths and relaxation times, Geophys. J. R. Astron. Soc., 59, 497-522.

Narteau, C., P. Shebalin, and M. Holschneider (2002), Temporal limits of the power law aftershock decay rate, J. Geophys. Res., 107(B12), 2359, doi:10.1029/2002JB001868.

Nur, A., and J. R. Booker (1972), Aftershocks caused by pore fluid flow?, Science, $175,885-888$

Riele, H. J. J. (1985), A program for solving first kind Fredholm integral equations by means of regularization, Comput. Phys. Commun., 36, 423-432.

Ripperger, J., and P. M. Mai (2004), Fast computation of static stress changes on 2D faults from final slip distributions, Geophys. Res. Lett., 31, L18610, doi:10.1029/2004GL020594.

Schaff, D. P., G. C. Beroza, and B. E. Shaw (1998), Postseismic response of repeating aftershocks, Geophys. Res. Lett., 25, 4549-4552.

Scholz, C. H. (1968), Microfractures, aftershocks, and seismicity, Seismol. Soc. Am. Bull., 58, 1117-1130.

Shaw, B. E. (1993), Generalized Omori law for aftershocks and foreshocks from a simple dynamics, Geophys. Res. Lett., 20, 907-910.

Toda, S., and R. Stein (2003), Toggling of seismicity by the 1997 Kagoshima earthquake couplet: A demonstration of time-dependent stress transfer, J. Geophys. Res., 108(B12), 2567, doi:10.1029/2003JB002527.

Toda, S., R. S. Stein, P. A. Reasenberg, and J. H. Dieterich (1998), Stress transferred by the $\mathrm{Mw}=6.5 \mathrm{Kobe}$, Japan, shock: Effect on aftershocks and future earthquake probabilities, J. Geophys. Res., 103, 24,543-24,565.

Toda, S., R. S. Stein, K. Richards-Dinger, and S. B. Bozkurt (2005), Forecasting the evolution of seismicity in southern California: Animations built on earthquake stress transfer, J. Geophys. Res., 110, B05S16, doi:10.1029/2004JB003415.

Ziv, A. (2003), Foreshocks, aftershocks, and remote triggering in quasi-static fault models, J. Geophys. Res., 108(B10), 2498, doi:10.1029/ 2002JB002318.

Ziv, A., and A. M. Rubin (2003), Implications of rate-and-state friction for properties of aftershock sequence: Quasi-static inherently discrete simulations, J. Geophys. Res., 108(B1), 2051, doi:10.1029/2001JB001219.

A. Helmstetter, Laboratoire de Géophysique Interne et Tectonophysique, Université Joseph Fourier, F-38041 Grenoble Cedex 9, France. (ahelmste@ obs.ujf-grenoble.fr)

B. E. Shaw, Lamont-Doherty Earth Observatory, 61 Route 9W, Palisades, NY 10964, USA. (shaw@1deo.columbia.edu) 